\title{
Reconstruction of flame temperature field with Optical Sectioning Method
}

\author{
Bin Zhou ${ }^{1}$ Jianyong Zhang ${ }^{2}$ Shimin Wang ${ }^{1}$ \\ ${ }^{1}$ Southeast University \\ Nanjing 210096, CHINA \\ zhoubinde@seu.edu.cn \\ ${ }^{2}$ University of Teesside \\ Middlesbrough TS1 3BA, UK
}

Abstract-The temperature information is of great significance to the study of flame structure, soot distribution and combustion mechanism. It is also vital to combustion control. This paper presents the design, implementation and evaluation of a flame imaging system based on optical sectioning method to reconstruct the steady state temperature field of candlelight. A 3-D luminous object can be regarded as a combination of a series of two dimensional luminous sections, hence, based on the superposition theorem, the original luminosity distribution of each section can be retrieved from the focused and the defocused images taken by adjusting the focus plane on each of the sections with a single CCD camera. From the relationship between the gray level of image and the temperature, the temperature profile of each section can be reconstructed. The experimental results prove the feasibility of the proposed method which can be used as a new measurement and diagnostic tool for quantitative characterization of steady state flame.

\section{Keywords- flame; temperature measurement; Optical Sectioning; image reconstruction}

\section{INTRODUCTION}

The temperature measurement has great significance to the study of the chemical reaction rate, combustion structure and chemical composition distribution of a flame. The flame visualization technology can be used to improve the combustion system design and to help understand the nature of combustion mechanism so that the optimal control can be achieved in terms of efficiency and emission.

Over the past two decades, there have been increased research activities in this particular area. Waterfall et al. measured the location of the flame front, flame size, air-fuel ratio, as well as the flame compositions of an internal combustion engine model using Electrical Capacitance Tomography [1]. This method offers high temporal resolution, and it can be used for online continuous monitoring. However, its measurement accuracy is limited due to the noise induced by the impact of flame sparks to the electrodes, and in some cases the variation in capacitance is insignificant to the flame change. Junta carried out experiments on instantaneous temperature distribution reconstruction inside the turbulent flame of a propane-air premix burner using multidirectional holographic interferogram [2]. Holographic interferometry flame CT gives high accuracy with a wide measurement range, but the disadvantages of this technique are the complexity of the optical system and the difficulty in stripe 
identification in the case with dense stripes. Brisley et al. also demonstrated a single camera system for the temperature measurement of a gaseous flame based on the cross-sectional reconstruction [3]. The single-camera approach operates on the assumption that the flame is axisymmetric in its shape and structure. Zhou et al. carried out the numerical and experimental investigations into the 3-D temperature distribution inside a large-scale coal-fired furnace using up to eight CCD cameras [4]. Knaus et al. proposed a new technique to acquire the data for instantaneous, high-resolution and thermal structure image reconstruction using crossed-plane Rayleigh imaging [5].

All these methods mentioned above have their unique features, however the complexity of structures and high costs limit their industrial applications. A simple, robust and reliable measurement system for the flame temperature distribution with the high spatial and temporal resolutions is required. Based on the theory of Optical Sectioning Microscopy (OSM), which was first recommended by Agard and Sedat in 1983 [6] for a 3-D fluorescence chromosome, a new system is proposed in this paper for flame temperature reconstruction by measuring the series of overlapped images of the flame sections. Based on the relationship between the luminosity and the temperature, the temperature distribution of flame can be reconstructed.

The paper is organized as follows. In Section II, the measurement principle of Optical Sectioning using a CCD is introduced, and then in Section III, the experimental setup of the optical system is presented. On the basis of the theoretical analysis, a novel method is proposed in section IV to determine the defocused Optical transfer function (OTF). In order to verify the feasibility of this method, the images of translucent films and thin sticks arranged along the axis are taken, and the quality of image reconstructions is evaluated. Furthermore, in section V, considering the characteristics of flame emissivity and absorptivity, the mathematical model is built. Based on the measurements of the monochromatic radiation absorption coefficient and the emissivity of the candle flame, the luminosity and temperature distribution of flame profiles are reconstructed respectively, which reveal the internal combustion structure and combustion characteristics of the candlelight. Finally, the main conclusions of this work are briefly given in Section VI.

\section{PRINCIPLE OF OpticAl SeCtioning Microscopy}

The combination of three-dimensional (3D) de-convolution with optical-sectioning microscopy was demonstrated more than two decades ago in the visionary work of Agard and Sedat [6]. As shown in Fig.1, the coordinates of the object space originates from the left boundary of the object, and axis z coincides with the optical axis. The coordinates of the image plane are denoted as ( $\mathrm{x}^{\prime}$, $\left.\mathrm{y}^{\prime}\right)$.

The monochromatic non-coherent light $\mathrm{f}(\mathrm{x}, \mathrm{y}, \mathrm{z})$ emits from a 3-D luminous object of thickness $\mathrm{T}$, and assume no energy absorption between different sections. 
When a camera focusing on plane $z^{\prime}$, the projection $g^{\prime}\left(x^{\prime}, y^{\prime}, z^{\prime}\right)$ can be regarded as the superposition of the images of the focused plane z' and other defocused planes. According to Fourier's optics theory, for a linear shift-invariant optical imaging system, the luminosity projection $\mathrm{g}_{1}\left(\mathrm{x}, \mathrm{y}, \mathrm{z}^{\prime}\right)$ on the imaging plane is the convolution of the luminosity function of the object $\mathrm{f}(\mathrm{x}, \mathrm{y}, \mathrm{z})$ and the Point Spread Function (PSF) h (x, y, z $\left.\mathrm{z}_{1} \mathrm{z}^{\prime}\right)$ of the optical system,

$$
g_{1}\left(x, y, z^{\prime}\right)=f(x, y, z) * h\left(x, y, z_{1}-z^{\prime}\right)
$$

If the plane $\mathrm{z}$ ' is focused on, the total luminosity distribution of the image is,

$$
g\left(x, y, z^{\prime}\right)=\int_{0}^{T} f(x, y, z) * h\left(x, y, z^{\prime}-z\right) d z
$$

After discretization, it is converted to:

$$
g\left(x, y, z^{\prime}\right)=\sum_{i=1}^{N} f(x, y, i \Delta z) * h\left(x, y, z^{\prime}-i \Delta z\right) \Delta z
$$

In the above formula, $N=T / \Delta z$ refers to the number of the sections of the object, each with the thickness of $\Delta z$. That is, the 3-D object can be considered as a combination of N-layer parallel two-dimensional profiles. Thus, the two dimensional images captured by a CCD camera contain 3-D information of a given luminous object.

Keeping the imaging system and the object on the same optical axis direction, a series of projection images can be captured when the camera is focused on the different cross-sections in sequence,

$$
g(x, y, j \Delta z)=\sum_{i=1}^{N} f(x, y, i \Delta z) * h(x, y, j \Delta z-i \Delta z) \Delta z, j=1,2, \cdots, N
$$

Equation (4) can be simplified as,

$$
g_{j}=\sum_{i=1}^{N} f_{i} * h_{j-i}, j=1,2, \cdots, N
$$

The above equation is usually Fourier transformed to,

$$
G_{j}\left(f_{x}, f_{y}\right)=\sum_{i=1}^{N} F_{i}\left(f_{x}, f_{y}\right) \times H_{j-i}\left(f_{x}, f_{y}\right), j=1,2, \cdots, N
$$

where $G, F$ are the Fourier transforms of $g, f$ respectively, and $H$ is the optical transfer function (OTF) of the optical system. $f_{x}$ and $f_{y}$ are the spatial frequency components. If the OTFs of the optical system at different defocused positions are known, equation (6) becomes closed and solvable. Finally, by performing inverse Fourier transform, the original luminosity distribution of each section $f_{j}(\mathrm{j}=1,2, \ldots, \mathrm{N})$ can be obtained,

$$
f_{j}(x, y)=\iint F_{i}\left(f_{x}, f_{y}\right) \exp \left[j 2 \pi\left(x f_{x}+y f_{y}\right)\right] d f_{x} d f_{y}, \quad j=1,2, \cdots, N
$$


Fig. 2 shows a schematic of the image formation and restoration chain, where ${ }^{*}$ stands for the convolution operation. In the forward image formation process, the acquired blurred image contaminated by noise is produced by the convolution of object and the PSF of the optical imaging system. To take the restoration quality and processing complexity into consideration, the task of image restoration is to give an estimate of the original image. The computational 3-D image deconvolution technique can be categorized into six broad classes: no-neighbors methods [7], neighboring methods [8], linear methods, nonlinear methods [9], statistical methods, and blind deconvolution methods. An overview of various deconvolution techniques on 3-D fluorescence microscopy images was detailed by Pinaki [10]. In this paper, the Tikhonov inverse-filtering method [11] is applied to image reconstruction, which is one of simplest and truest linear 3-D deconvolution methods.

\section{EXPERIMENTAL SETUP}

The experimental setup is shown in Fig.3 (a), the lenses and the color CCD camera can be moved forward and backwards at 0.8 millimeter of each pitch on a slider horizontally by turning the screw driven by a stepper motor. The electric motor rotates at rev/400-pulse, and thus the resolution of the axial movement is $2 \mu \mathrm{m}$. The vertical position of the system can also be adjusted to make the center of the lens align with the object. The internal configuration of the lenses system (Gco2302-2304) is shown in Fig. 3 (b). It is a combination of front and back afocal lenses with the aperture stops located at the common focus of the front and back lenses, resulting in the chief rays being parallel to the optical axis in both object and image spaces, i.e. both the entrance and exit pupils are at infinity. A constant perspective or viewing angle and a constant magnification can therefore be achieved in the cases when the object and image surfaces are tilted with respect to the optical axis or the lens is defocused on either object surface or image surface. The depth of field of the lenses is about 1 millimeter.

The control flow diagram of the system is shown in Fig.4. After initialization of the system parameters including the focus of lens, aperture, OTF, section number $\mathrm{N}$ and the interval $\Delta \mathrm{Z}$, and then images are captured in sequence by the CCD camera and stored for each $\Delta \mathrm{Z}$. When the set number of images is reached, the optical system returns to its original position.

\section{IMAGE RECONSTRUCTIONS OF TRANSLUCENT FILMS AND THIN STICKS USING OPTICAL SECTIONING METHOD}

The images of a series of translucent films along the same axis are reconstructed to confirm the feasibility of the method.

\section{A. Calibration of PSF and OTF}

In a system with a single CCD camera, the images of different colours may be synchronously obtained either on a monochrome sensor array through a beam splitting /filtering assembly with narrowband interference filters [12], or on a colour sensor array with a mosaic pixel filter (for example, red-green-blue (RGB)) Bayer filter) [13, 14]. The CCD sensor used in this experiment is the later. 
The RGB narrowband measurement is simplified as three effective wavelength $\left(\lambda_{R}, \lambda_{G}, \lambda_{B}\right)$ measurements. The monochromatic measurement in $\mathrm{R}$ is used in the calculation, and $\lambda_{\mathrm{R}}$ is chosen to be $625 \mathrm{~nm}$ according to the spectrum response of the color CCD provided by the manufacturer.

From equation 6, it can be seen that OTFs of the system need to be determined before the equations can be solved. There are several ways to determine OTF [15-17]. For example, Fourier transform of a blurred image may allow the zeros in the OTF to be seen [18]. However, it is not known if there is any coincide of zeros between $F\left(f_{x}, f_{y}\right)$ and $H\left(f_{x}, f_{y}\right)$. Therefore, the authors propose a priori knowledge based method to find the single-parametric OTF, which has been proven to be reliable.

Since the defocused OTF can be expressed in Stokseth approximation formula [19]:

$$
\begin{aligned}
& H_{s}(\omega, w)=2\left(1-0.69 \omega+0.0076 \omega^{2}+0.043 \omega^{3}\right) \frac{J_{1}(a-0.5 a \omega)}{(a-0.5 a \omega)}, \\
& a=(4 \pi / \lambda) w \omega
\end{aligned}
$$

where $\mathrm{w}$ denotes the optical path difference out of focus. $\lambda$ is the wavelength. $\omega$ is the frequency. Under the given defocused distance, the OTF is determined by the value of $\mathrm{w}$ which can restore the image well from equation 8 . For the optical system mentioned above, the OTF and PSF of $2 \mathrm{~mm}$ defocused distance are shown in Fig. 5 where $\mathrm{w} / \lambda=0.26$.

\section{B. Experiments on reconstruction of translucent films}

In Fig. 6 (a), three translucent films are arranged along the axial direction with $2 \mathrm{~mm}$ interval apart. Focusing on each film in sequence, the gray level distributions of R from the captured superimposed images are shown in Fig. 6 (b). Fig. 6 (c) shows the reconstructed image using Tikhonov inverse-filtering method [11], and it can be seen that the inversion result is satisfactory and the effect of superposition has been partially eliminated.

Defining the similarity correlation coefficient of two images as following [20-21],

$$
\frac{\sum_{i=1}^{m} \sum_{j=1}^{n} a(i, j) \times b(i, j)}{\sqrt{\sum_{i=1}^{m} \sum_{j=1}^{n} a(i, j)^{2} \times \sum_{i=1}^{m} \sum_{j=1}^{n} b(i, j)^{2}}}
$$

where $a(i, j)$ and $b(i, j)$ are pixel gray level of $R$ in image, $m, n$ for the pixel number of row and column respectively. Comparing the inversion result with the original films using equation (9), the correlation coefficients are $0.916,0.813$ and 0.957 respectively. 


\section{Experiments on reconstruction of thin sticks}

In Fig. 7 (a), five $0.5 \mathrm{~mm}$ diameter sticks were arranged in three rows with $2 \mathrm{~mm}$ interval between them. The superimposed images and the reconstructed image are show in Fig. 7 (b) and (c) respectively. The correlation coefficients are $0.912,0.935$ and 0.961 respectively.

There are two possible reasons for an object to emit light, i.e. reflection and self-emitting irradiance. The reflected irradiance contains the information on the reflectivity or refractive index of an object, whilst the self-emitting irradiance carries the information about the spectral emissivity of a luminous object. If a luminous body is the flame instead of translucent films or thin sticks, it is obvious that each profile of the flame can also be reconstructed based on the method described above.

\section{RECONSTRUCTION OF PROFILE TEMPERATURE DISTRIBUTION OF FLAME}

It is essential to find the relationship among the absorption characteristics of the flame radiation, flame absorptivity, the monochromatic emissivity, image gray and temperature before the temperature distribution of a flame can be reconstructed using OST.

\section{A. Characteristics of emissivity and absorptivity of flame}

The thermal radiation produced by the fuel combustion is electromagnetic waves, the spectrum of which covering the visible region is captured by the $\mathrm{CCD}$ camera in this system. Ignoring the relative small radiation of combustion gases $\left(\mathrm{CO}, \mathrm{CO}_{2}, \mathrm{H}_{2} \mathrm{O}\right.$, etc.), approximately, the luminous radiation is the radiation of high temperature soot cloud caused by the incomplete combustion of fuel [22].

The soot cloud is an absorptive-emissive medium, which emits its own energy outwards and simultaneously absorbs energy from external sources. The scattering effects of soot may be negligible assuming the size of particle is much smaller than the wavelength of radiation. Therefore, the attenuation of ray intensity caused by the absorption of soot cloud is [23],

$$
d i_{\lambda}=-\alpha_{\lambda} i_{\lambda} d s
$$

The integration of the above equation gives,

$$
i_{\lambda}(s)=i_{\lambda}(0) \exp \left(-\alpha_{\lambda} s\right)
$$

where $s$ is the thickness of soot cloud. $i_{\lambda}(0)$ is the intensity of monochromatic radiation of the incident ray. $i_{\lambda}(s)$ is the intensity of monochromatic radiation of the transmitted ray. $\alpha_{\lambda}$ is the monochromatic absorption coefficient of the soot cloud.

And the average monochromatic transmissivity is, 


$$
\tau_{\lambda}(s)=\frac{i_{\lambda}(s)}{i_{\lambda}(0)}=\exp \left(-\alpha_{\lambda} s\right)
$$

So the average monochromatic absorptivity is,

$$
\hat{a}_{\lambda}=1-\exp \left(-\alpha_{\lambda} s\right)
$$

According to Kirchhoff's law, the average monochromatic emissivity $\bar{\varepsilon}_{\lambda}$ is equal to the average monochromatic absorptivity $\hat{a}_{\lambda}$ in the thermal equilibrium state,

$$
\bar{\varepsilon}_{\lambda}=\hat{a}_{\lambda}=1-\exp \left(-\alpha_{\lambda} s\right)
$$

Fig. 8 shows the schematic of the soot cloud with uniform concentration. When a ray with an initial intensity $i_{\lambda}\left(s_{n-1}\right)$ penetrates a distance $\Delta$ through the cloud, assuming $\alpha_{\lambda}$ unchanged in $\Delta$, the radiation from the outside surface of $s_{n}$ is the sum of the transmissive and emissive radiation, thus

$$
i_{\lambda}\left(s_{n}\right)=i_{\lambda}\left(s_{n-1}\right) \exp \left(-\alpha_{\lambda} \Delta\right)+\bar{\varepsilon}_{\lambda} \cdot i_{b \lambda}\left(s_{n}\right)
$$

where $\mathrm{i}_{\mathrm{b} \lambda}$ is the emission intensity of the blackbody. If the soot cloud is divided into n sections, each with the same thickness $\Delta$, we have,

$$
i_{\lambda}\left(s_{n-1}\right)=i_{\lambda}\left(s_{n-2}\right) \exp \left(-\alpha_{\lambda} \Delta\right)+\bar{\varepsilon}_{\lambda} i_{b \lambda}\left(s_{n-1}\right)
$$

Thus, from equations (12) and (13), the radiation from the surface of the last section is,

$$
i_{\lambda}\left(s_{n}\right)=i_{\lambda}\left(s_{n-2}\right) \exp \left(-\alpha_{\lambda} 2 \Delta\right)+\bar{\varepsilon}_{\lambda} i_{b \lambda}\left(s_{n-1}\right) \exp \left(-\alpha_{\lambda} \Delta\right)+\bar{\varepsilon}_{\lambda} i_{b \lambda}\left(s_{n}\right)
$$

From equations (13) and (14), it yields,

$$
i_{\lambda}\left(s_{n}\right)=\sum_{k=1}^{n} \bar{\varepsilon}_{\lambda} i_{b \lambda}(k \Delta) \exp \left(-\alpha_{\lambda}(n-k) \Delta\right)
$$

Form the above analysis, it can be concluded that the radiation from 3-D flame (soot cloud) can be seen as the weighted sum of the radiation from a series of two-dimensional luminous sections. The received radiation contains the information of the 3-D radiation of the flame.

\section{B. Measurements of average monochromatic absorption coefficient and average emissivity}

From equation (15), it can be seen that the unknown parameters $\bar{\varepsilon}_{\lambda}$ and $\alpha_{\lambda}$ need to be determined before solving this equation. There are many methods for measuring absorption coefficient and emissivity, e.g. the monochromatic radiation and absorption method [24], the radiation absorption integral method, the multiple spectral reflexion method [25] and the temperature-color method [26]. The monochromatic radiation and absorption method is used here. 
A reference semiconductor laser is placed at the left side of the flame in Fig. 9, which produces a monochromatic radiation at the wavelength of $625 \mathrm{~nm}$. The light signal was detected by a photodiode after it penetrates the flame and the $625 \mathrm{~nm}$ narrowband interference filter.

Measurement procedures are as follows:

- $\quad$ Laser source only without flame, measure the output signal $\mathrm{L}_{\mathrm{s}}$;

- Flame only without the laser source, measure the output signal $\mathrm{L}_{\mathrm{f}}$;

- Measure the output signal L when the laser passes through the flame, therefore we have

$$
\begin{aligned}
& L=L_{s}\left(1-\hat{a}_{\lambda}\right)+L_{f} \\
& \hat{a}_{\lambda}=\frac{L_{s}+L_{f}-L}{L_{s}}=1-\exp \left(\alpha_{\lambda} s\right) \\
& \alpha_{\lambda}=\frac{\operatorname{In}\left(1-\hat{a}_{\lambda}\right)}{s}
\end{aligned}
$$

Assuming uniform absorption coefficient, the absorption coefficient of the flame can be calculated from the above equations. Experimental results are given in Table 1.

TABLE I. EXPERIMENTAL MEASUREMENTS OF FLAME ABSORPTION COEFFICIENT $(\mathrm{s}=10 \mathrm{~mm})$

\begin{tabular}{cccccc}
\hline No. & $\mathrm{L}_{\mathrm{s}}(\mathrm{mv})$ & $\mathrm{L}_{\mathrm{f}}(\mathrm{mv})$ & $\mathrm{L}(\mathrm{mv})$ & $\hat{\mathrm{a}}_{\lambda}$ & $\alpha_{\lambda}(1 / \mathrm{mm})$ \\
\hline 1 & 59 & 9390 & 9431 & 0.305 & 0.0364 \\
2 & 61 & 9396 & 9439 & 0.295 & 0.0350 \\
3 & 60 & 9392 & 9435 & 0.283 & 0.0333 \\
4 & 60 & 9395 & 9437 & 0.283 & 0.0333 \\
5 & 59 & 9395 & 9436 & 0.305 & 0.0364 \\
6 & 62 & 9394 & 9438 & 0.290 & 0.0342 \\
7 & 61 & 9394 & 9438 & 0.279 & 0.0327 \\
8 & 60 & 9391 & 9434 & 0.283 & 0.0333 \\
Average & & & & 0.290 & 0.0343
\end{tabular}

For $\mathrm{s}=10 \mathrm{~mm}$, the average value of $\hat{\mathrm{a}}_{\lambda}\left(\bar{\varepsilon}_{\lambda}\right)$ is $0.290, \alpha_{\lambda}=0.0343 / \mathrm{mm}$, the transmissivity of flame of different thickness $\mathrm{n} \Delta$ is given in table 2 .

TABLE II. TRANSMISSIVITY OF FLAME OF DIFFERENT THICKNESS N $\Delta$

\begin{tabular}{|l|l|l|l|l|l|}
\hline $\mathrm{n} \Delta(\mathrm{mm})$ & 1 & 2 & 3 & 4 & 5 \\
\hline
\end{tabular}




\begin{tabular}{|c|c|c|c|c|c|}
\hline $\exp \left(-\mathrm{n} \Delta \alpha_{\lambda}\right)$ & 0.966 & 0.934 & 0.902 & 0.872 & 0.842 \\
\hline $\mathrm{n} \Delta(\mathrm{mm})$ & 6 & 7 & 8 & 9 & 10 \\
\hline $\exp \left(-\mathrm{n} \Delta \alpha_{\lambda}\right)$ & 0.814 & 0.787 & 0.760 & 0.734 & 0.710 \\
\hline
\end{tabular}

\section{Mathematical modeling and calibration of optical transfer function}

From the above analysis, it can be seen that the 3-D flame can be regarded as a combination of a series of flame sections. This is, the radiation from 3-D flame is the superposition sum of the radiation from different flame sections. When the $j_{\text {th }}$ section is focused on, the received luminosity on CCD is,

$$
\begin{aligned}
& L_{j}=\bar{\varepsilon}_{\lambda} \cdot i_{b \lambda}(\Delta) * h_{1-j} \exp \left(-\alpha_{\lambda}(n-1) \Delta\right)+ \\
& \bar{\varepsilon}_{\lambda} \cdot i_{b \lambda}(2 \Delta) * h_{2-j} \exp \left(-\alpha_{\lambda}(n-2) \Delta\right)+ \\
& \text {... }(j \Delta) * h_{0} \exp \left(-\alpha_{\lambda} \Delta\right)+ \\
& \text {... }
\end{aligned}
$$

where $L_{j}$ is radiation projected on the CCD array. $h_{n-j}$ is the PSF of the defocused distance $(n-j) \Delta$.

Based on the proposed measurement method introduced in section IV.A, the PSFs/OTFs in equations (17) can be obtained using the thin film fixed at different defocused distance. For example, in Fig.10 the photos of the films captured at different defocused distance $0 \mathrm{~mm}$ (on focus), $2 \mathrm{~mm}, 4 \mathrm{~mm}, 6 \mathrm{~mm}, 8 \mathrm{~mm}, 10 \mathrm{~mm}$ can be retrieved well when $\mathrm{w}=0,0.27 \lambda, 0.52 \lambda, 0.77 \lambda, 1.02 \lambda, 1.27 \lambda$ respectively, where $\lambda=625 \mathrm{~nm}$. Accordingly, the defocused PSF can be determined, as shown in Fig. 11.

In Fig. 12, some parts of the reconstructed images are enlarged to present the details.

\section{Relationship between the gray level image and the temperature of the flame}

The radiation of the flame travels through the optical system and projects onto the sensing area of CCD detector. The induced charge, proportional to the luminosity, are collected and converted to the voltage signal which is further quantified by graphic card. According to the Planck's Law, in visible light wavelength, the relationship between the monochromatic luminosity $\mathrm{L}_{\lambda}$ of flame and its temperature can be expressed in [27],

$$
L_{\lambda}=\varepsilon(\lambda, T) \frac{c_{1}}{\pi \lambda^{5}} \frac{1}{e^{c_{2} / \lambda T}-1}
$$

where $C_{1}$ and $C_{2}$ are the Planck constants, $\lambda$ is the wavelength, the relationship between image gray level and the luminosity is as follows [28]:

$$
g=\frac{1}{u_{\max }-u_{\min }}\left(2^{a^{*}}-1\right) 2^{b^{*}} \cdot \eta_{3} \cdot\left[\frac{\tau}{4} \pi L_{\lambda}(D / f)^{2} A t \eta_{1} \eta_{2}-u_{\min }\right]
$$


where $\mathrm{D} / \mathrm{f}$ is the relative aperture, $\mathrm{A}$ is the sensing area of CCD array, $\mathrm{u}_{\max }$ is the maximum voltage level of the luminosity, $\mathrm{u}_{\mathrm{min}}$ is the minimum voltage level of the luminosity, $\eta_{1}$ is quantum efficiency, $\eta_{2}$ is transfer efficiency and $\eta_{3}$ is transfer coefficient. a ${ }^{*}$ and $\mathrm{b}^{*}$ are digitized bits. $\mathrm{t}$ is the exposure time and $\tau$ is the light transmittance of the lenses.

If $\mathrm{u}_{\min }$ can be ignored (very small), equation (19) is simplified to,

$$
g=\Phi t(D / f)^{2} L_{\lambda}
$$

Where $\Phi$ is the instrument constant. The instrument constant can be eliminated if the equation (20) is transformed into,

$$
\frac{g}{g_{\text {ref }}}=\frac{L_{\lambda}}{L_{\lambda r e f}}
$$

where $\mathrm{g}_{\text {ref }}$ and $\mathrm{L}_{\mathrm{ref}}$ mean the reference gray level and luminosity respectively. It can be seen that the relative luminosity distribution can be determined from the gray level image.

The emissivity $\varepsilon$ is the function of wavelength $\lambda$ and temperature T. After the wavelength $\lambda$ has been fixed, the monochromatic emissivity $\varepsilon_{\lambda}$ is the function of temperature T. For soot particle characteristic of continuous radiation, the emissivity function can be approximately written in the series form [29],

$$
\varepsilon_{\lambda}(T)=\sum_{i=0}^{m} b_{i} T^{i}
$$

Combining equations (18), (21) and (22), we have,

$$
\frac{g}{g_{\text {ref }}}=\frac{L_{\lambda}}{L_{\lambda \text { ref }}}=\frac{\sum_{i=0}^{m} b_{i} T^{i}}{\sum_{i=0}^{m} b_{i} T_{\text {ref }}^{i}} \frac{e^{c_{2} / \lambda T_{\text {ref }}}-1}{e^{c_{2} / \lambda T}-1}
$$

The unknown temperature coefficients $b_{i}(i=0, \ldots, m)$ can be determined by $n=m+2$ practical temperature measurements. $m$ is chosen as 3 by experience here. When the relationship between the emissivity and the temperature is determined, the temperature distribution can be solved from the gray level image.

\section{E. Reconstruction results of flame and discussions}

The photo of the experimental setup is shown in Fig.13. A steady candlelight stands in front of the lens. Two methods can be used to prevent the saturation caused by the over high luminosity of the flame, 1 . Put an attenuation filter between the flame and the lenses or 2. Adjust the exposure time of CCD. It is necessary to mention that the axial resolution up to $\mu \mathrm{m}$ can be achieved by OST. Fig.14 shows the results for five sectioning to the candlelight. Higher axial resolution would add too much burden on reconstruction calculations. Due to the limited view field of camera, the upper and lower areas of the flame are measured and calculated separately. 
The captured (R) gray level image of the lower part of the candlelight is shown in Fig. 15 (a). There seems no apparent difference between these images. However, the normalized luminosity distributions in Fig. 15 (b), which can be regarded as the superimposition of the degraded luminosity distributions of different sections, are quite different from the deconvolution results in Fig. 15 (d). Furthermore, the reconstructed gray level images in Fig.15 (c) are different form each other, the area of the inner section of the flame is larger than that of the outer. The normalized luminosity distributions of the reconstructed gray level images given in Fig. 15(d) reflect the structure of radiation and the combustion inside the candlelight.

In Fig. 15 (e), the temperature of the bottom part of the candlelight is comparatively low. It is known that the radiation in this part is primarily in the infrared region, which is not used as measurements in this work. With the devolatilization of paraffin wax, more smoke particles are formed, resulting in higher temperature in the middle and upper parts of the flame. The temperature reaches its maximum value at the combustion surface and decreases towards inner region filled with mixture of combustible and diffusion products and towards outside region filled with mixture of exhaust gases and air. Due to the relationship between the monochromatic luminosity $\mathrm{L}_{\lambda}$ of the flame and its temperature given in equation (18), the variation of luminosity in Fig. 15 (d) is more intensive than the variation of temperature in Fig. 15 (e). Practically, the variation of luminosity can reflect the variation of the flame radiation better.

The points temperature measurements are acquired using an S-type thermocouple of $0.125 \mathrm{~mm}$ of diameter, which is connected to a data logger. The position of the thermocouple's tip can be accurately controlled by a specially designed positioning mechanism. Based on the relationship between the emissivity and the temperature, the temperature distribution can be calculated by solving equation (23). Comparing the temperature in the 3rd picture of Fig. 15(e) with that measured by the thermocouple in Fig. 16, it is obvious that the temperature distribution is very similar. The inversion results of the gray level, normalized luminosity and temperature distribution of the upper area of the candlelight are presented in Fig. 17. It can be seen that the diameter of the combustion region shrinks along the altitude of the candlelight as the fuel is running out. The phenomenon of unburned carbon emerging from the top of the flame is also observable.

Although the candlelight is assumed to be steady in the measurement process, it is difficult to achieve in practice, as the shape and structure of the flame have been changing. If the speed of adjusting the optical system along the height of the flame is not fast enough, the luminosity and temperature distributions given in upper and lower sections can not be considered to belong to the same flame. Therefore, an optical system with large field of view and fast adjustment speed is desirable for the implementation of the proposed method. 


\section{CONCLUSIONS}

A prototype measurement system based on Optical Sectioning method has been developed for reconstruction of steady state flame temperature field. The inversion results of the candlelight reflect the distribution of flame internal radiation, the distribution of temperature, as well as the combustion structure of candlelight, which verifies this method. Only one fixed position CCD camera is required. The system's configuration is simple and the method is reliable. This measurement system is for offline use. The time cost for one shot is $10 \mathrm{~ms}$. The repositioning speed is $50 \mathrm{~mm} / \mathrm{s}$ and the distance between each section is $2 \mathrm{~mm}$. So the total time cost for one cycle is $0.21 \mathrm{~s}$. The future work will be focused on using the electronic-controlled zoom of liquid lens to replace the stepping motor, which will significantly increase the adjustment speed of focus plane. An efficient inversion algorithm is needed to overcome the difficulty of reconstructions of more sectioning cases. It is envisioned that the system will be a useful tool for investigation of the underlying physics of the flame evolution and emission formation.

\section{ACKNOWLEDGEMENTS}

The authors would like to express their gratitude to the Major Program of National Natural Science Foundation of China (No.50836003), to the Science Foundation of Ministry of Education of China for the New Teacher (No. 20090092120064), to the National Natural Science Foundation for youths (No. 50906013).

\section{REFERENCES}

[1] R. C. Waterfall, R. He, and C. M. Beck.: 'Visualizing combustion using electrical impedance tomography’ , Chem. Eng. Sci., 1997, 52,(13),pp. 2129-2138

[2] Junta Doi, Seishiro Sato.: '3-D modeling of the instantaneous temperature distribution in a turbulent flame using a multidirectional interferometer', Optical Engineering., 2007, 46,(1), pp.015601-1-015601-7

[3] P. B. Brisley, G. Lu, Y. Yan, and S. Cornwell.: 'Three dimensional temperature measurement of combustion flames using a single monochromatic CCD camera', IEEE Trans. Instrum.Meas., 2005,54, (4), pp.1417-1421

[4] H. C. Zhou, C. Lou, Q. Cheng, Z. Jiang, J. He, B. Huang, Z. Pei, and C. Lu.: 'Experimental investigations on visualization of 3-D temperature distributions in a large-scale pulverized-coal-fired boiler furnace', Proceedings of the Combustion Institute.,2005,30, pp.1699-1706

[5] D.A. Knaus, S.S. Sattle, F.C. Gouldin.: '3-D temperature gradients in premixed turbulent flame lets via crossed-plane Rayleigh imaging', Combustion and Flame., 2005 141,(3) ,pp.253-270

[6] Agard D. A., Sedat J W.: '3-D Architecture of a Polytene Nucleus',Nature., 1983 ,302, pp.676-681

[7] J.G. McNally, T.Karpova, J.Cooper, and J.A. Conchello.: 'Three-dimensional imaging by deconvolution microscopy', Methods., 1999,19, (3),pp.373-385

[8] D.A.Agard.: 'Optical sectioning microscopy: Cellular architecture in three dimensions', Ann.Rev.Biophys.Bioeng., 1984 ,13,pp.191-219

[9] P.J. Verveer and T.M.Jovin.: 'Accleration of the ICTM image restoration algorithm',J.Micro., 1997, 188, pp.191-195

[10] Sarder,P. Nehorai,A.: 'Deconvolution methods for 3-D fluorescence microscopy images',Signal Processing Magazine,IEEE.,2006 ,23,pp.32-45 
[11] A.N. Tikhonov and V.Y.Arsenin.: 'Solution of Ill-Posed Problems'(Wiley: New York., 1977)

[12] G. Lu and Y. Yan.: 'Temperature profiling of pulverized coal flames using multicolor pyrometric and digital imaging techniques', IEEE Trans. Instrum. Meas., 2006,.55,(4), pp.1303-1308

[13] T. R. Fu, X. F. Cheng, C. L. Shi, M. H. Zhong, T. M. Liu, and X. B. Zheng.: 'The setup of a vision pyrometer', Meas. Sci. Technol., 2006 ,17, pp.659-665

[14] Z. X. Luo and H. C. Zhou.: 'A combustion-monitoring system with 3-D temperature reconstruction based on flame-image processing technique',IEEE Trans. Instrum. Meas., 2007,56,(5), pp1877-1882

[15] J. Flusser, T. Suk.: 'Degraded image analysis, an invariant approach', IEEE Trans. Pattern Analysis and Machine Intelligence., 1998 ,20, (6), pp. 590-603

[16] M. Cannon.: 'Blind deconvolution of spatially invariant image blurs with phase', IEEE Trans. On Acoustics, Speech and Signal Processing, 2000 , ASSP-24, (1), pp.58-63

[17] D. Kundur, D. Hatzinakos.: 'Blind image deconvolution revisited', IEEE Signal Processing Magazine., 1996,13,(6), pp.61-63

[18] D. Gennery.: 'Determination of optical transfer function by inspection of frequency domain plot', Journal of the Optical Society of America., 1973, 63, pp.1571-1577

[19] Stokseth P.A.: ' Properties of a defocused optical system', Journal of the Optical Society of America., 1969,59, (10), pp.1314-1321

[20] W. Fang. ' nonlinear image reconstruction algorithm for electrical capacitance tomography', Meas .Sci. Technol., 2004,15 pp. $2124-2132$

[21] W.Q. Yang, L.H. Peng.: ‘Image reconstruction algorithms for electrical capacitance tomography’, Meas .Sci. Technol., 2003,14,pp. R1-13

[22] S. H. Qian.: ‘Principle of Gas Combustion’(China Architectural Press, 1989)

[23] N. L. Wang.: 'Optical measurement technology of particle and its application'( Atomic Energy Press, 2000)

[24] Lu Y. C., Freyman T. M., Hernandez G, Kuo K K.: 'Measurement of temperatures and OH concentrations of solid propellant flames using absorption spectroscopy', Combustion Science and Technology.,1995 ,104,pp.193-205

[25] Chen Y.: 'A method for measuring emissivities and true temperatures from multiple spectral reflexion in pyrometry', High Temperature-High Presures., $1992,24,(1), \mathrm{pp} 75-85$

[26] Zhu de zhong.: 'Laser measurement technology in thermal engineering'(Science press,1990)

[27] Zhou H. C.: 'Detection theory and technology of Flame visualization in Furnace'(Science press ,2005)

[28] Yong gang Lu, Shimin Wang.:‘3-Dimensional Irradiance Reconstruction with a Single Camera system’, Journal of Southeast University, 1998,14,(2), pp.3842

[29] Tairan Fu.: 'Principle study and technique application of primary spectrum pyrometry', PhD thesis, University of Science and Technology of China, 2005

\section{FIGURES AND LIST OF FIGURE CAPTIONS}

Figure 1. Optical system schematic of OST

Figure 2. Schematic of the image formation and restoration chain

Figure 3. (a) Schematic of the experimental setup b) Configuration of the lenses system

Figure 4. Control flow diagram of the system

Figure 5. Optical Transfer Function and Point Spread Function under 2mm defocused distance

Figure 6. Reconstruction results of translucent films

Figure 7. Reconstruction results of five sticks 
Figure 8. Schematic of the soot cloud and sectioning

Figure 9. Scheme of measurement of flame absorption coefficient

Figure 10. Photos under different defocused distance of (1) 0mm (focus); (2) 2mm; (3) 4mm; (4) 6mm; (5) 8mm; (6) 10mm

Figure 11. PSFs under different defocused distance of (1) 2mm; (2) 4mm; (3) 6mm; (4) 8mm; (5) 10mm (z coordinates for luminosity)

Figure 12. Reconstructed images under different defocused distance of (1) 2mm, (2) 4mm, (3) 6mm, (4) 8mm, (5) 10mm

Figure 13. Photo of the experimental setup

Figure 14. Different parts of the candlelight

Figure 15. Reconstruction of lower part of the candlelight

Figure 16. Temperature measurements in the middle section of candlelight using the thermocouple

Figure 17. Reconstruction of upper part of the candlelight

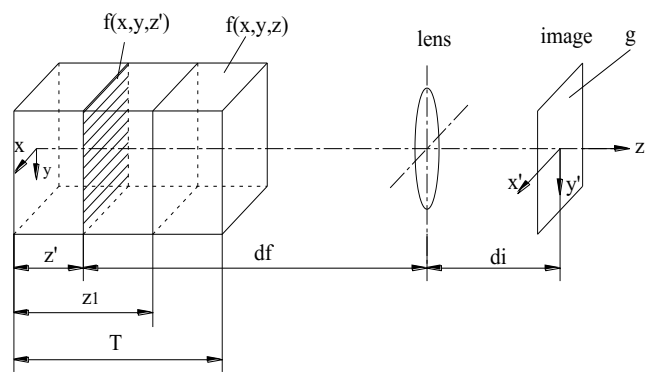

Figure 1. Optical system schematic of OST

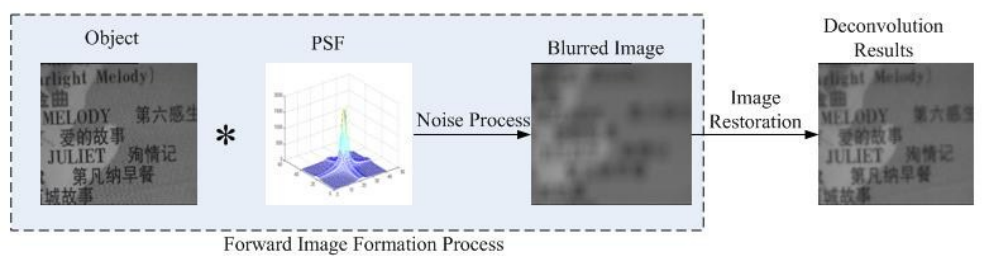

Figure 2. Schematic of the image formation and restoration chain

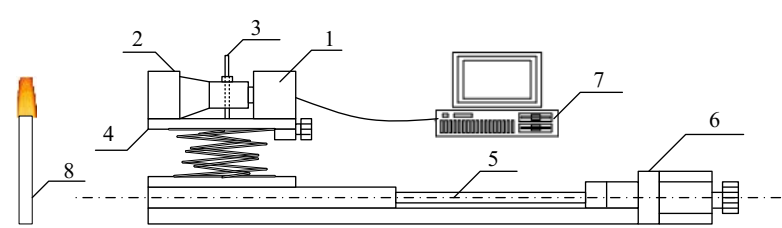

1- CCD camera; 2- Lenses; 3- Adjustment mechanism;

4 - Scissor type lift; 5 - Screw; 6 - Stepper motor; 7 - Computer; 8 - Object

(a)

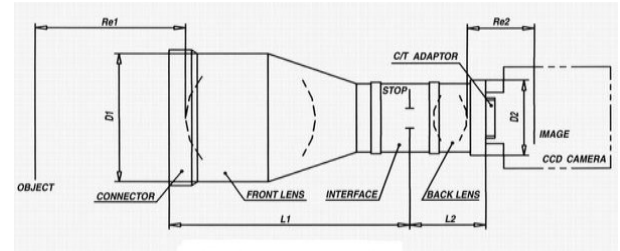

(b)

Figure 3. Schematic of the experimental setup and Configuration of the lenses system 


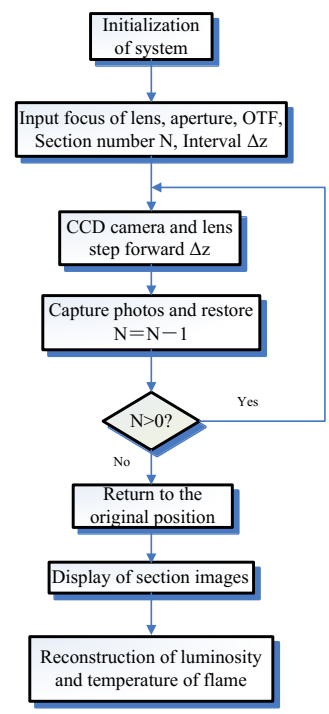

Figure 4. Control flow diagram of the system

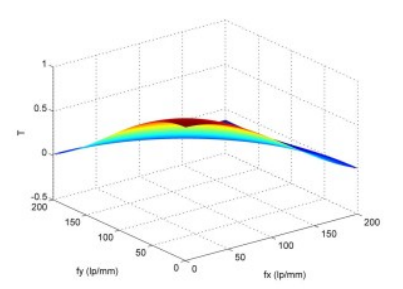

(a) OTF

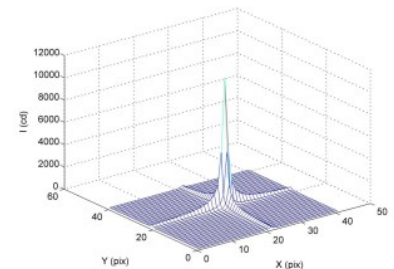

(b) PSF

Figure 5. Optical Transfer Function and Point Spread Function under $2 \mathrm{~mm}$ defocused distance 


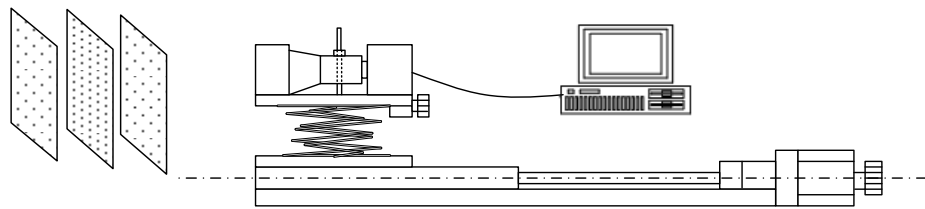

(a) Translucent films arrangement

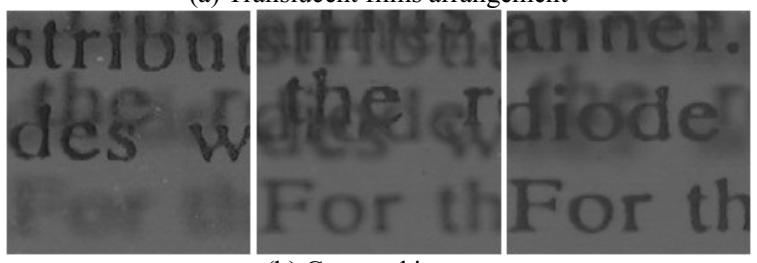

(b) Captured images

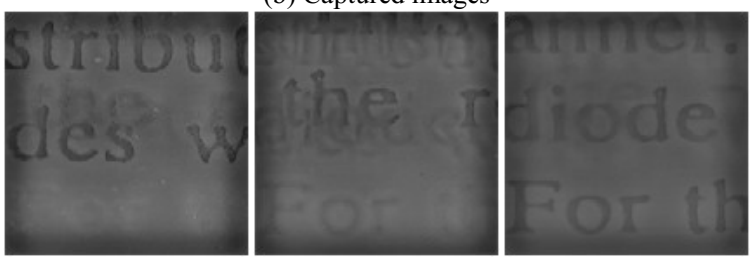

(c) Reconstructed images

Figure 6. Reconstruction results of translucent films 


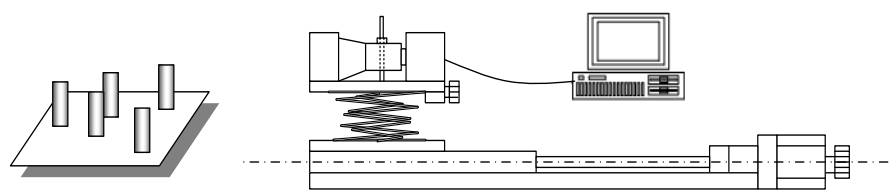

(a) Five sticks arrangement

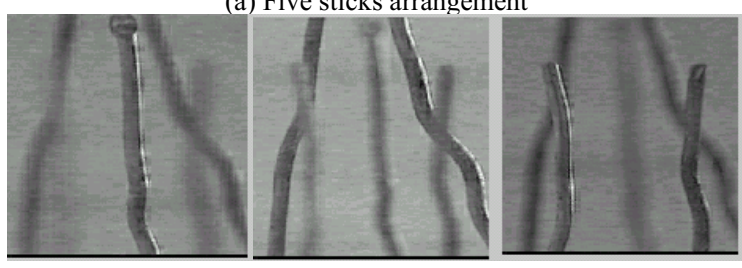

(b) Captured images

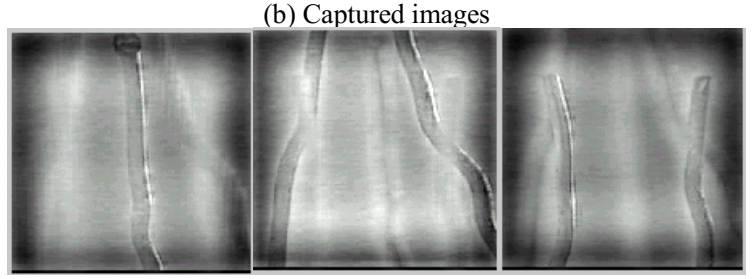

(c) Reconstructed images

Figure 7. Reconstruction results of five sticks

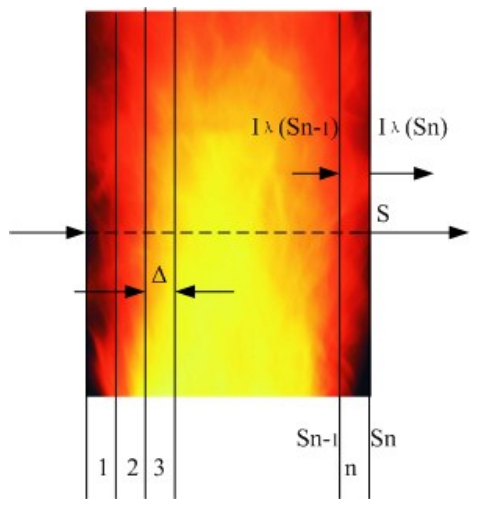

Figure 8. Schematic of the soot cloud and sectioning

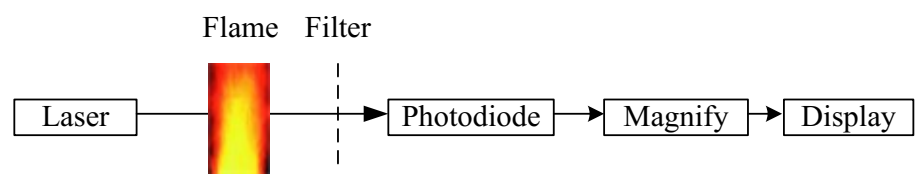

Figure 9. Scheme of measurement of flame absorption coefficient 


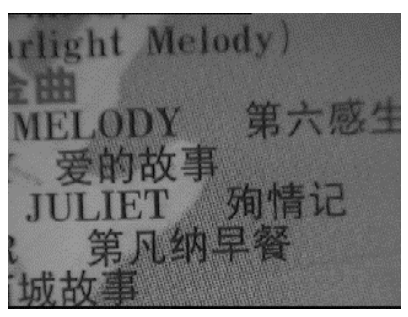

(1)

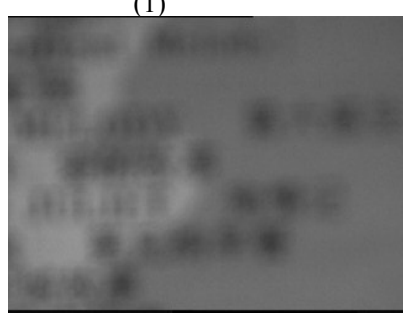

(4)

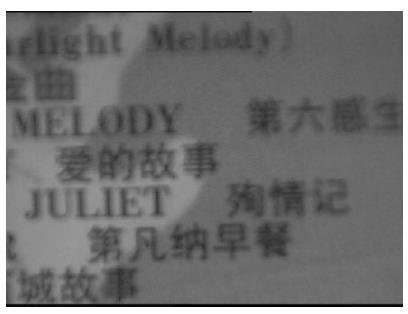

(2)

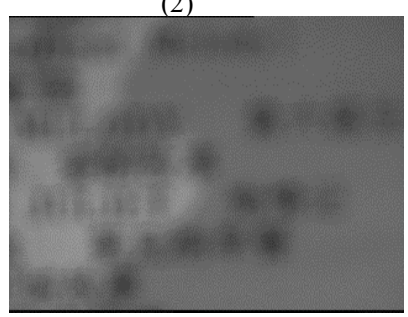

(5)

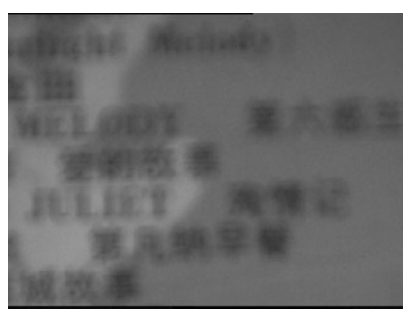

(3)

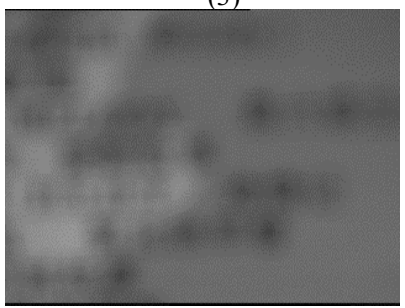

(6)

Figure 10. Photos under different defocused distance of (1) $0 \mathrm{~mm}$ (focus); (2) 2mm; (3) 4mm; (4) 6mm; (5) 8mm; (6) 10mm

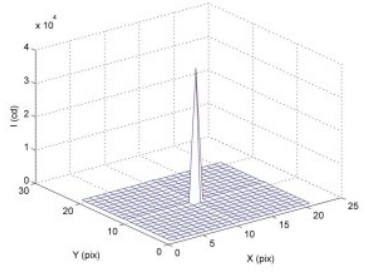

(1)

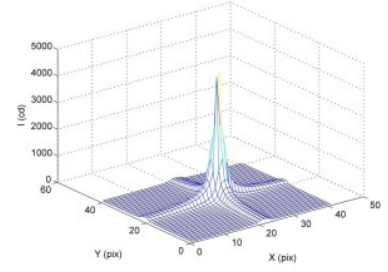

(2)

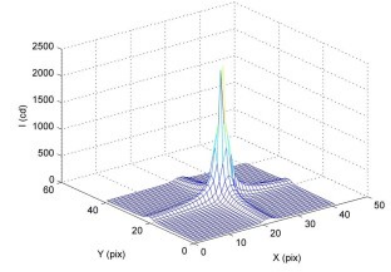

(3)

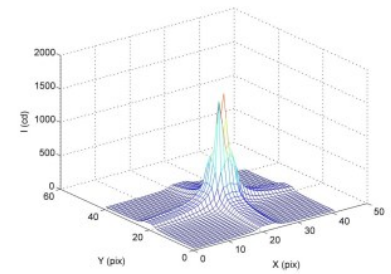

(4)

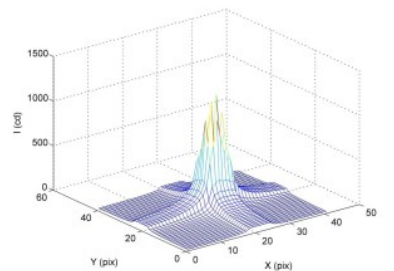

(5)

Figure 11. PSFs under different defocused distance of (1) 2mm; (2) 4mm; (3) 6mm; (4) 8mm; (5) 10mm (z coordinates for luminosity) 


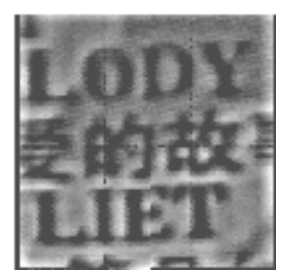

(1)

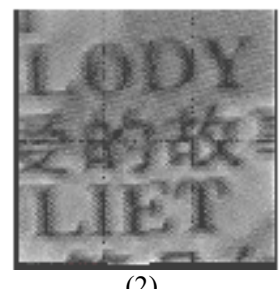

(2)

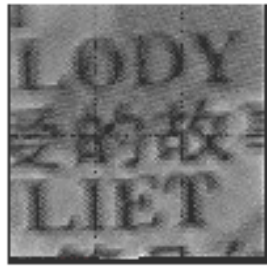

(3)

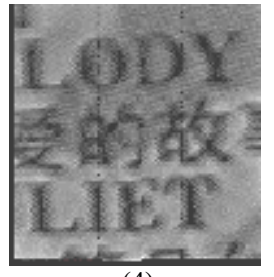

(4)

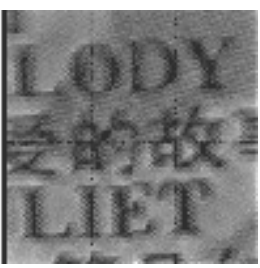

(5)

Figure 12. Reconstructed images under different defocused distance of (1) $2 \mathrm{~mm}$, (2) $4 \mathrm{~mm}$, (3) $6 \mathrm{~mm}$, (4) $8 \mathrm{~mm}$, (5) $10 \mathrm{~mm}$

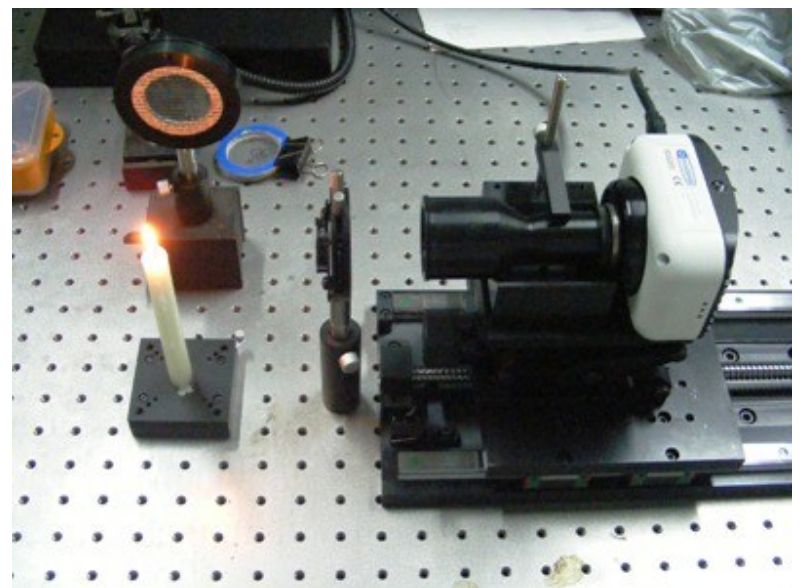

Figure 13. Photo of the experimental setup
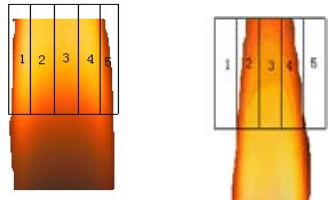

(a)Lower part of the flame (b) Upper part of the flame

Figure 14. Different parts of the candlelight 

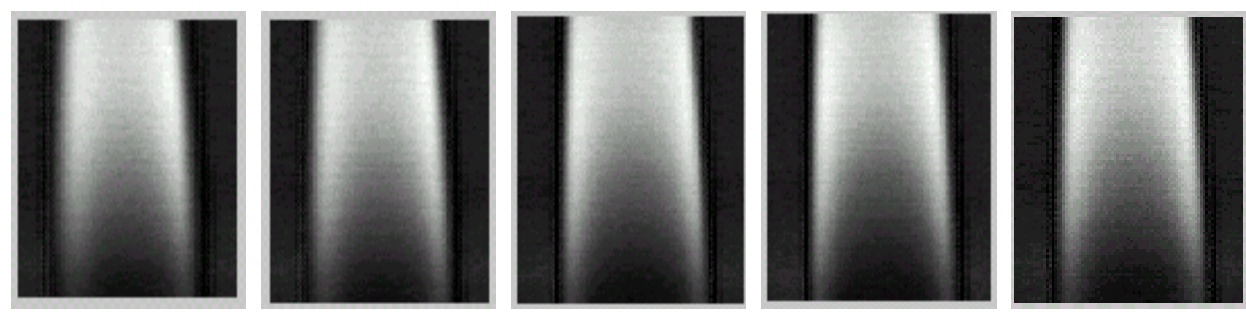

(a) Gray level images captured by CCD camera for different longitudinal sections
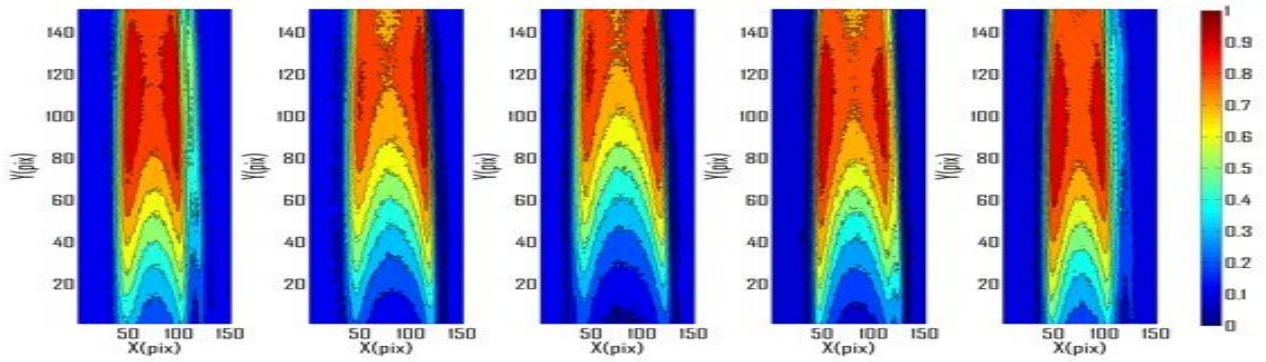

(b) Normalized luminosity distributions for different longitudinal sections
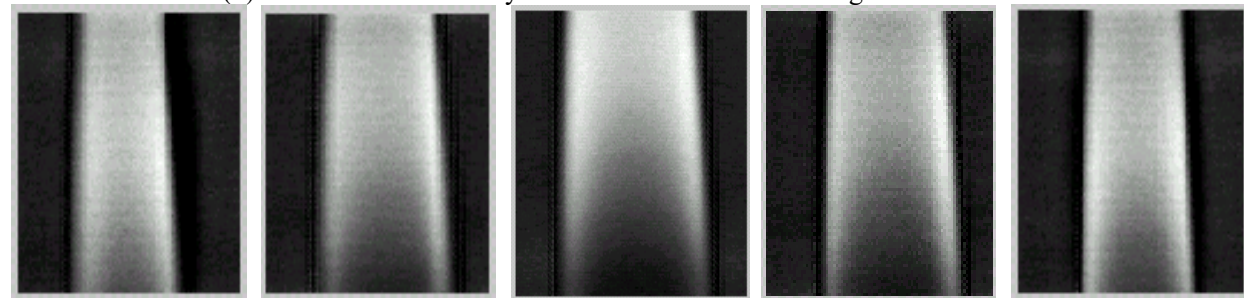

(c) Reconstructed gray level images for different longitudinal sections
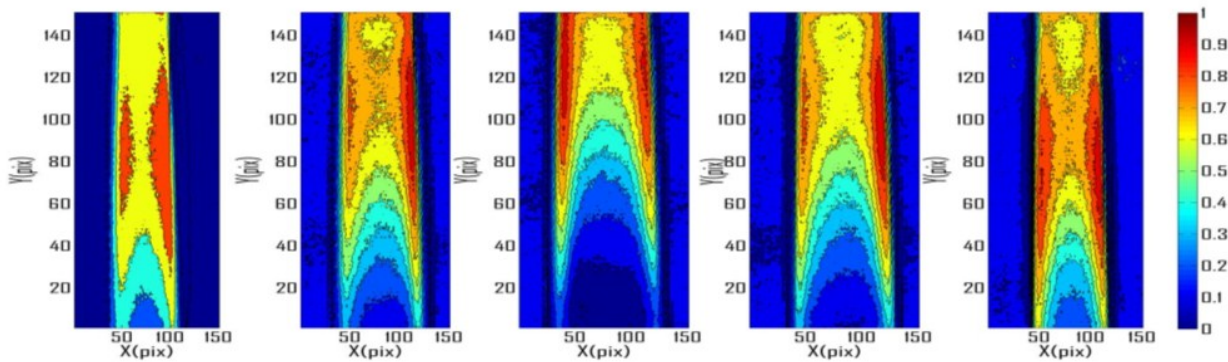

(d) Reconstructed normalized luminosity for different longitudinal sections
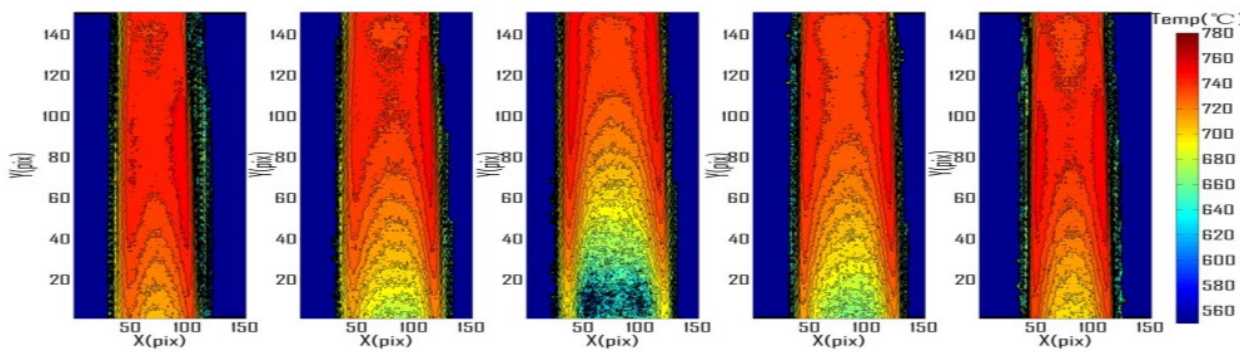

(e) Reconstructed temperature distributions for different longitudinal sections

Figure 15. Reconstruction of lower part of the candlelight 


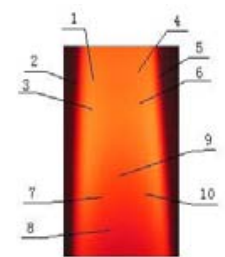

$1-735^{\circ} \mathrm{C} ; 2-618^{\circ} \mathrm{C} ; 3-728^{\circ} \mathrm{C} ; 4-739^{\circ} \mathrm{C} ; 5-613^{\circ} \mathrm{C}$

$6-720^{\circ} \mathrm{C} ; 7-685^{\circ} \mathrm{C} ; 8-612^{\circ} \mathrm{C} ; 9-672^{\circ} \mathrm{C} ; 10-668^{\circ} \mathrm{C}$

Figure 16. Temperature measurements in the middle section of candlelight using the thermocouple 

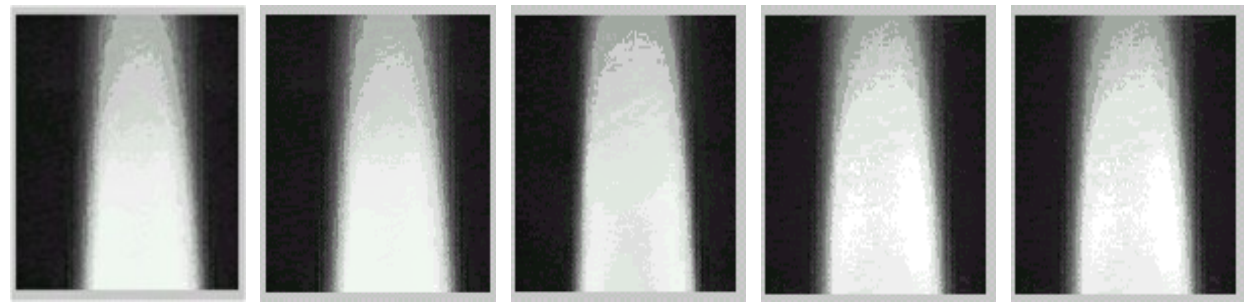

(a) Gray level images captured by CCD camera for different longitudinal sections
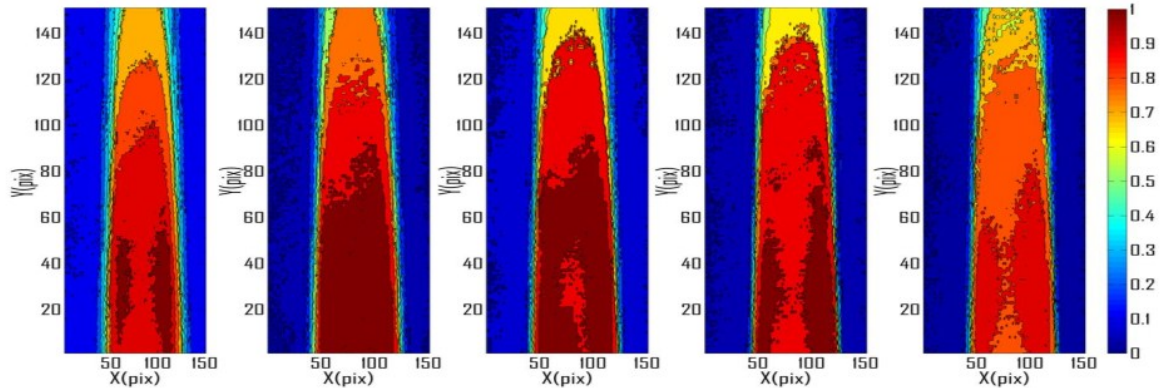

(b) Normalized luminosity distributions for different longitudinal sections
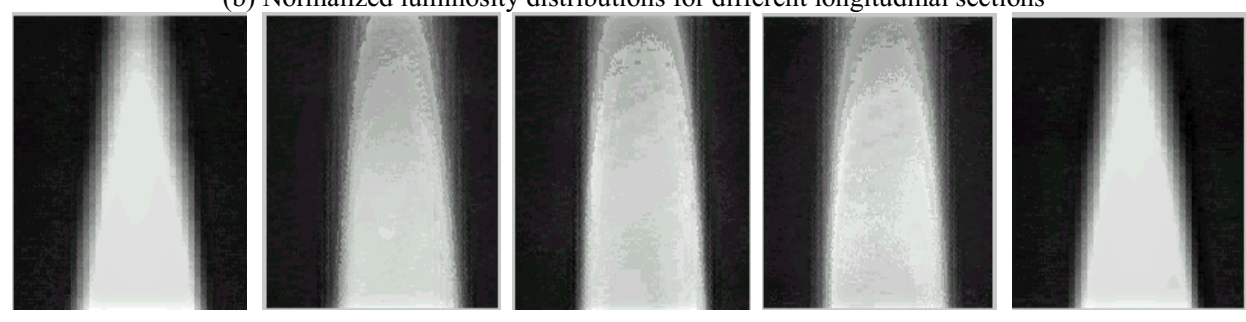

(c) Reconstructed gray level images for different longitudinal sections
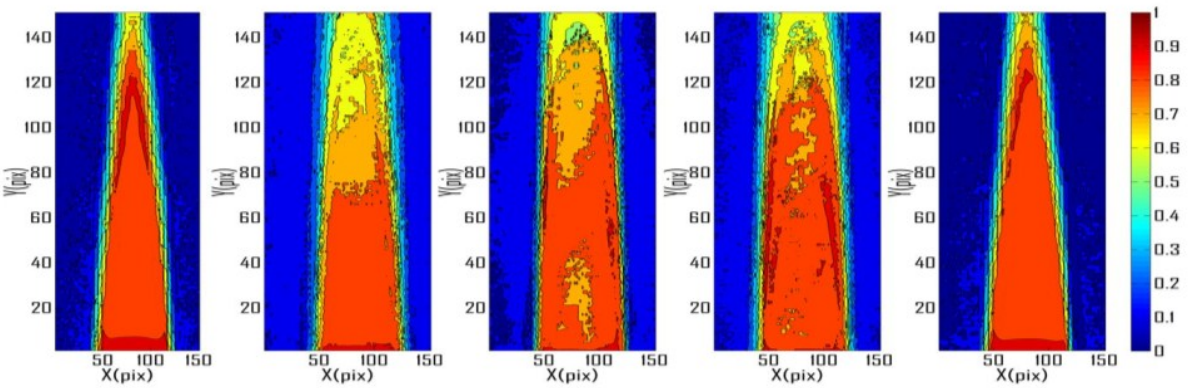

(d) Reconstructed normalized luminosity for different longitudinal sections
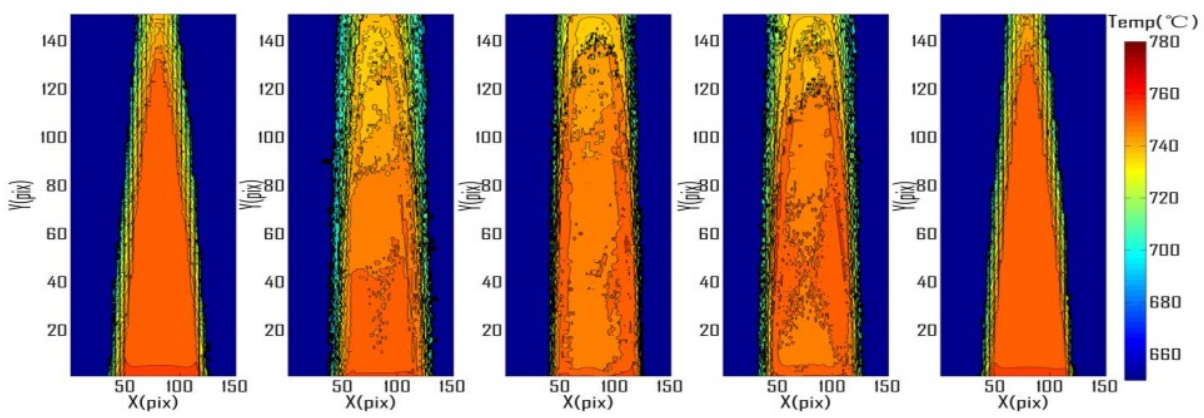

(e) Reconstructed temperature distributions for different longitudinal sections

Figure 17. Reconstruction of upper part of the candlelight 